\title{
Association of p53 codon 72 polymorphism with susceptibility to hepatocellular carcinoma in a Chinese population from northeast Sichuan
}

\author{
JIAJING CAI ${ }^{1-3 *}$, YAN CAI $^{1-3 *}$, QIANG MA ${ }^{1-3}$, FAN CHANG $^{3}$, LEI XU $^{3}$, \\ GUOYUAN ZHANG ${ }^{1,2}$ and XIAOLAN GUO ${ }^{1-3}$
}

${ }^{1}$ Department of Laboratory Medicine, North Sichuan Medical College; ${ }^{2}$ Department of Clinical Laboratory, Affiliated Hospital of North Sichuan Medical College; ${ }^{3}$ Translational Medicine Research Center, North Sichuan Medical College, Nanchong, Sichuan 637000, P.R. China

Received November 4, 2016; Accepted December 7, 2016

DOI: $10.3892 /$ br. 2017.840

\begin{abstract}
The p53 tumor suppressor gene is key in tumor development and progression, and the single nucleotide polymorphism (SNP) of the p53 gene codon 72 (p53Arg/Pro) changes the structure of the protein. In addition, it affects its activity, which may affect cancer risk. The aim of the present study was to investigate the association between p53 codon 72 polymorphism and susceptibility to hepatocellular carcinoma (HCC) in a Chinese population from northeast Sichuan. A total of $342 \mathrm{HCC}$ patients and 347 non-cancer control subjects were recruited, and the polymorphism of p53 codon 72 was measured by TaqMan ${ }^{\circledR}$ minor groove binder fluorescent quantitative polymerase chain reaction assay. The distribution frequency of p53 sites of arginine (Arg)/Arg, Arg/proline (Pro), Pro/Pro were 18.4, 48.8 and $32.8 \%$ in the control group, as compared with $18.7,49.9$ and $31.4 \%$ in the case group, which indicated that there was no difference between two groups $\left(\chi^{2}=0.14 ; \mathrm{P}=0.93\right)$. Upon further stratification with smoking, alcohol consumption, gender and hepatitis B virus (HBV) infection, no risk increasing genotype was identified. However, interactions between p53 codon 72 SNP and smoking, alcohol consumption and HBV infection may increase the risk of HCC [smoking odds ratio (OR), 2.00; 95\% confidence interval (CI), 1.21-3.29; alcohol consumption OR, 1.87; 95\% CI, 1.08-3.26; HBV infection OR, 1.84; 95\% CI, 1.10-3.08]. No significant association was identified
\end{abstract}

Correspondence to: Professor Xiaolan Guo, Department of Clinical Laboratory, Affiliated Hospital of North Sichuan Medical College, 63 Wenhua Road, Nanchong, Sichuan 637000, P.R. China E-mail: alan5200@hotmail.com

Abbreviations: HCC, hepatocellular carcinoma; SNP, single nucleotide polymorphism; HBV, hepatitis B virus

${ }^{*}$ Contributed equally

Key words: hepatocellular carcinoma, p53, single nucleotide polymorphism, susceptibility between p53 codon 72 polymorphism and $\mathrm{HCC}$, and it may not have an independent effect on the susceptibility to HCC in a Chinese population from northeast Sichuan. However, interaction between genetic factors and environment exposure significantly increased the risk of HCC.

\section{Introduction}

Hepatocellular carcinoma (HCC) is commonly referred to as 'the king of cancer'. The latest data indicate that the morbidity and mortality of HCC in China accounts for 50.5 and $51.4 \%$ cases worldwide, respectively (1). In Sichuan, China, the incidence of HCC ranks second only to lung cancer (2). Previous studies have shown that the major risk factors contributing to $\mathrm{HCC}$ in China were chronic hepatitis B virus (HBV) infection and aflatoxin B1 exposure (3). Other associated risk factors include hepatitis $\mathrm{C}$ virus infection, smoking and alcohol consumption. However, HCC develops only in a small group of HBV carriers, implying that when exposed to the same environmental conditions, genetic factors may contribute to the carcinogenic mechanism.

p53 is one of the most investigated tumor suppressor genes and mutations of $\mathrm{p} 53$ have been confirmed in $>50 \%$ of human tumors (4). A guanine (G)/cytosine (C) common single nucleotide polymorphism (SNP) at p53 codon 72, results in the replacement of an arginine (Arg) by proline (Pro). No significant difference was identified between the two polymorphic variants regarding the function, structure and stability in most cells. Furthermore, the Pro allele possesses a stronger ability to activate transcription, and the Arg variant inhibits transformed cell growth and induces apoptosis more effectively (5). Whether the protein mutation due to SNP is involved in hepatocarcinogenesis remains inconclusive. Currently, to the best of our knowledge, there is no study regarding the association between p53 codon 72 polymorphism and HCC susceptibility in a Chinese population from northeast Sichuan, where HCC-associated morbidity and mortality are particularly high (2). The current case-control study was performed to address this issue, and to investigate the interaction between $\mathrm{p} 53$ codon 72 genotypes and environmental factors in the development of HCC. 


\section{Materials and methods}

Study population. The present study was a hospital-based case-control study. The case group included 342 HCC patients, the mean age was $55.53 \pm 11.80$ years, among which 295 cases were male and 47 were female; the control group was composed of 347 cases of cancer-free patients, the mean age was $53.21 \pm 13.17$ years, with 301 males and 46 females. All subjects were recruited from the Affiliated Hospital of North Sichuan Medical College (Nanchong, China) from January 2014 to January 2015. The HCC patients were treated at the Affiliated Hospital of North Sichuan Medical College from January to July 2014 and all patients were confirmed by pathological diagnosis. The control subjects were recruited from the same hospital during the same period and had no family history of cancer. General characteristics, such as age, gender, smoking, alcohol consumption and hepatitis B surface antigen ( $\mathrm{HBsAg}$ ) were collected by querying medical records. All subjects provided written informed consent and this study was approved by the Ethics Committee of North Sichuan Medical College.

DNA extraction. Genomic DNA was extracted from 200- $\mu 1$ samples of peripheral blood using a column blood genome extraction kit (cat. no. DP318; Tiangen Biotech Co., Ltd., Beijing, China) according to the manufacturer's instructions. The extracted genomic DNA was stored at $-20^{\circ} \mathrm{C}$ in a refrigerator.

Genotyping. The p53 codon 72 polymorphism was measured using a TaqMan ${ }^{\circledR}$ minor groove binder fluorescent quantitative polymerase chain reaction (PCR) assay and Applied Biosystems SNP Genotyping Assay Mix (cat. no. rs1042522; Thermo Fisher Scientific, Inc., Waltham, MA, USA). The SNP Genotyping Assay Mix contained forward and reverse primers, and two hydrolysis probes labeled with FAM and VIC, respectively. The $5^{\prime} \rightarrow 3^{\prime}$ sequence of the primers was not provided. Endpoint SNP genotyping assays use hydrolysis probes for SNP genotyping. Two sequence-specific probes detect the wild-type and mutant alleles. Each is labeled with a FAM dye and a VIC dye, and fluorescence data are collected using PCR. To identify genotypes, only endpoint fluorescence intensities of the two reporter dyes are used and the relative dye intensities discriminate between homozygous for allele $\mathrm{x}$, homozygous for allele y, and heterozygous. A LightCycler 96 real-time fluorescent quantitative PCR instrument was also used (Fig. 1).

The PCR reaction system $(20 \mu \mathrm{l})$ consisted of the following: $2 \mathrm{X}$ TaqMan ${ }^{\circledR}$ Universal PCR mix $(10 \mu \mathrm{l}), 40 \mathrm{X}$ SNP Genotyping Assay mix $(0.5 \mu \mathrm{l})$, template DNA $(1 \mu \mathrm{l})$ and $\mathrm{H}_{2} \mathrm{O}(8.5 \mu \mathrm{l})$, and the following cycling conditions were used: $95^{\circ} \mathrm{C}$ for $10 \mathrm{~min}$ followed by 45 cycles of $95^{\circ} \mathrm{C}$ for $10 \mathrm{sec}$ and $60^{\circ} \mathrm{C}$ for $60 \mathrm{sec}$, with a final stage at $37^{\circ} \mathrm{C}$ for $30 \mathrm{sec}$.

Quality control. A deionized water reaction system served as a negative control. The genotyping experiments were conducted in double-blind conditions. For the failure samples, repeated testing, subsequent to establishing the reasons for failure, combined with PCR results excluded the samples without results following duplicate detection. A total of $10 \%$ of the samples were randomly selected for repeat testing and the results were $100 \%$ concordant.
Statistical analysis. The data were analyzed using SPSS 16.0 statistical software (SPSS, Inc., Chicago, IL, USA). Differences in the distributions of demographic characteristics between the cases and controls were evaluated using Student's t-test (for continuous variables) and the $\chi^{2}$ test (for categorical variables). The $\chi^{2}$ goodness-of-fit test was performed to calculate the distribution of p53 codon 72 in each genotype in the control group and to establish Hardy-Weinberg genetic equilibrium. The associations between p53 Arg72Pro genotypes and the risk of HCC were estimated by computing the odds ratios (ORs) and their 95\% confidence intervals (CIs) from multivariate logistic regression analysis. $\mathrm{P}<0.05$ was considered to indicate a statistically significant difference.

\section{Results}

General subject characteristics. No obvious differences in age and gender were identified between the control and case groups, while smoking, alcohol consumption and HBsAg status were statistically significant in these two groups (Table I; $\mathrm{P}<0.05$ ).

p53 codon 72 polymorphism and risk of HCC. Distribution of the genotypes among the control group was consistent with that expected from the Hardy-Weinberg equilibrium model $\left(\chi^{2}=0.06 ; \mathrm{P}=0.80\right)$. The results demonstrated that the distribution frequency of p53 sites of Arg/Arg, Arg/Pro, Pro/Pro were $18.4,48.8$ and $32.7 \%$, respectively, in the HCC group, and $18.7,49.9$ and $31.4 \%$, respectively, in the control group, which indicated that there were no obvious differences between the two groups $\left(\chi^{2}=0.14 ; \mathrm{P}=0.93\right.$; Table II).

Stratified analysis. To further establish whether the effect of genetic variation was modified by environmental factors, the HCC patients and control subjects were stratified by smoking status, alcohol consumption, gender and HBV infection; however, no differences in the distribution frequency of $\mathrm{p} 53$ sites were identified between the two groups. In addition, no association between the 553 codon 72 polymorphism and HCC susceptibility and environmental factors, such as smoking, alcohol consumption and HBV status, as well as gender was identified (Table III).

Interaction analysis of gene polymorphisms and environmental factors. The individuals who were not exposed to genetic and environmental risk factors were regarded as the reference when calculating ORs and 95\% CIs in the present study. The results demonstrated that interactions between p53 codon 72 polymorphism and environmental risk factors (smoking: OR, 2.00 and 95\% CI, 1.21-3.29; alcohol consumption: OR, 1.87 and 95\% CI, 1.08-3.26; HBV infection: OR, 1.84 and $95 \%$ CI, 1.10-3.08, but no gender) may increase the risk of HCC (Table IV).

\section{Discussion}

More than 700,000 new cases of HCC are diagnosed annually around the world, presenting a significant threat to human health and life (6). Early diagnosis of HCC is considered to be the most effective method of curing or controlling the progress of the patients, which prompts investigation of 

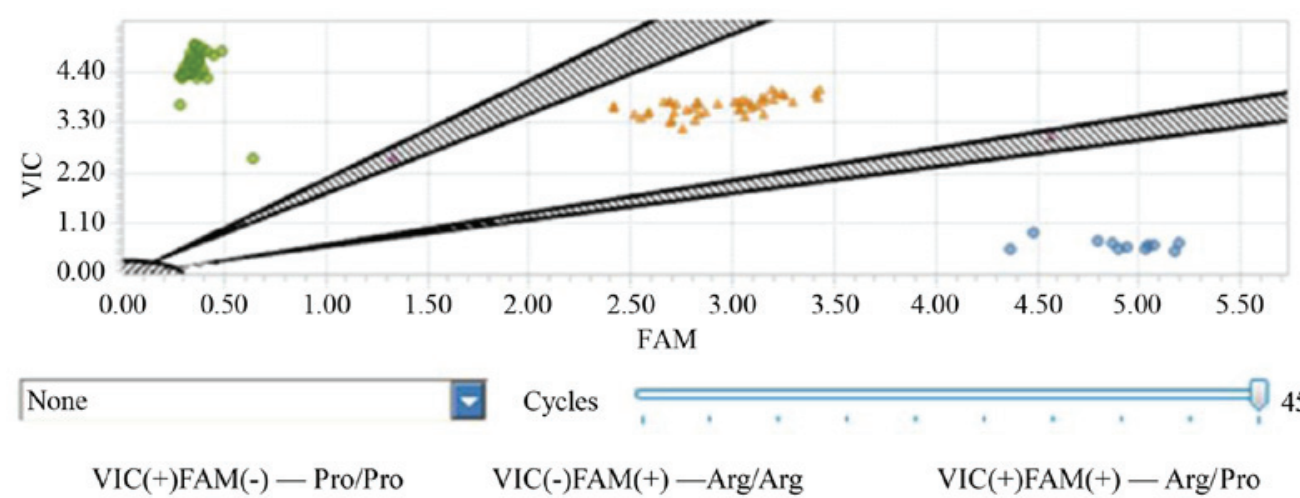

Figure 1. Genotyping of p53 codon 72 polymorphism. Two sequence-specific probes were used to detect the wild-type and mutant alleles. Allele C was labeled with VIC and allele G was labeled with FAM. VIC(+)FAM(-) indicates Pro/Pro homozygous, VIC(-)FAM(+) indicates Arg/Arg homozygous and VIC(+) FAM(+) indicates Arg/Pro heterozygous. Pro, proline; Arg, arginine.

Table I. Distribution of general characteristics in HCC patients and control subjects (means \pm standard deviation).

\begin{tabular}{lccc}
\hline Characteristics & $\begin{array}{c}\text { HCC } \\
(\mathrm{n}=342)\end{array}$ & $\begin{array}{c}\text { Control } \\
(\mathrm{n}=347)\end{array}$ & P-value \\
\hline $\begin{array}{l}\text { Age (years) } \\
\text { Gender }\end{array}$ & $55.53 \pm 11.80$ & $53.21 \pm 13.17$ & $>0.05^{\mathrm{a}}$ \\
$\quad \begin{array}{l}\text { Male } \\
\text { Female }\end{array}$ & 295 & 301 & \\
$\begin{array}{l}\text { Smoking history } \\
\text { Yes }\end{array}$ & 47 & 46 & $>0.05^{\mathrm{b}}$ \\
$\quad$ No & 163 & 121 & \\
$\begin{array}{l}\text { Alcohol } \\
\text { consumption }\end{array}$ & 179 & 226 & $<0.05^{\mathrm{b}}$ \\
$\quad$ Yes & & & \\
$\quad$ No & 206 & 154 & \\
$\begin{array}{l}\text { Hepatitis B } \\
\text { surface antigen }\end{array}$ & 136 & 193 & $<0.05^{\mathrm{b}}$ \\
+ & & & \\
- & 184 & 140 & \\
\hline
\end{tabular}

${ }^{\text {aa }}$ Student's t-test and ${ }^{\text {bPearson's }} \chi^{2}$ test. HCC, hepatocellular carcinoma.

the pathogenesis of HCC, to establish the risk factors and/or molecular markers for early diagnosis, which would be the pivotal. The aim of the current study was to establish the association between $\mathrm{p} 53$ codon 72 polymorphism and HCC in Sichuan, China and investigate whether the p53 codon 72 polymorphism may present as a potential non-invasive marker to predict the risk of HCC and/or to achieve early diagnosis of HCC.

The tumor suppressor gene p53 locates on chromosome 17p13.1, contains 11 exons and 10 introns, and encodes 53-kDa nuclear phosphoprotein. Many types of stress activate p53, for example proto-oncogene activation, DNA damage and hypoxia. As a transcription factor, p53 activates the expression of p21 and affects the cell cycle G1 phase checkpoints, regulates the cells entering the $S$ phase and manages the repair
Table II. Allele and genotype frequency distribution of p53 codon 72 polymorphism between the two groups.

\begin{tabular}{lrrrr}
\hline Category & HCC & Control & P-value & OR (95\% CI) \\
\hline Allele & & & & \\
Arg & 126 & 130 & & \\
Pro & 558 & 564 & 0.88 & $1.02(0.78-1.34)$ \\
Genotype & & & & \\
Arg/Arg & 63 & 65 & & \\
Arg/Pro & 167 & 173 & 0.98 & $1.00(0.66-1.50)$ \\
Pro/Pro & 112 & 109 & 0.79 & $1.06(0.69-1.64)$ \\
Dominant model & & & & \\
Arg/Arg & 63 & 65 & & \\
Arg/Pro + Pro/Pro & 279 & 282 & 0.92 & $1.02(0.70-1.50)$ \\
Recessive model & & & & \\
Arg/Arg + Arg/Pro & 230 & 238 & & \\
Pro/Pro & 112 & 109 & 0.71 & $1.07(0.77-1.46)$ \\
\hline
\end{tabular}

HCC, hepatocellular carcinoma; OR, odds ratio; CI, confidence interval; Arg, arginine; Pro, proline.

of damaged cells. When the repair fails, apoptosis is induced to prevent damage being passed on to offspring cells and ultimately suppresses tumorigenesis (7). Conversely, mutated p53 eventually results in cancer by impairing the injury stress reaction pathways. The Arg allele induces apoptosis more effectively, which may be due to the ability of localizing in mitochondria and regulating the release of cytochrome $c$ into cytosol. Cytochrome $c$ activates caspase-3, which is a pivotal executioner of apoptosis (8). Simultaneously, Arg suppresses colony formation of transformed cells, while the Pro variant exerts a stronger transcriptional activity. Different biological functions of the two proteins affect cell cycle regulation, DNA repair, apoptosis and tumorigenesis, which may be associated with the risk of carcinogenesis.

Various studies have attempted to define the association between p53 codon 72 polymorphism and HCC susceptibility; however, the findings were controversial and inconclusive with the different ethnic backgrounds of the study population 
Table III. Stratified analysis of the association between the p53 codon 72 polymorphism and risk of HCC.

\begin{tabular}{|c|c|c|c|c|c|}
\hline Characteristics & Genotype & $\mathrm{HCC}$ & Control & P-value & OR $(95 \% \mathrm{CI})$ \\
\hline \multicolumn{6}{|l|}{ Smoking history } \\
\hline \multirow[t]{2}{*}{ No } & Arg/Arg & 33 & 55 & & \\
\hline & Arg/Pro + Pro/Pro & 146 & 171 & 0.15 & $1.42(0.88-2.31)$ \\
\hline \multirow[t]{2}{*}{ Yes } & Arg/Arg & 30 & 10 & & \\
\hline & Arg/Pro + Pro/Pro & 133 & 111 & 0.02 & $0.40(0.19-0.85)$ \\
\hline \multicolumn{6}{|c|}{ Alcohol consumption } \\
\hline \multirow[t]{2}{*}{ No } & Arg/Arg & 26 & 36 & & \\
\hline & Arg/Pro + Pro/Pro & 110 & 157 & 0.92 & $0.97(0.55-1.70)$ \\
\hline \multirow[t]{2}{*}{ Yes } & Arg/Arg & 37 & 29 & & \\
\hline & Arg/Pro + Pro/Pro & 169 & 125 & 0.83 & $1.06(0.62-1.82)$ \\
\hline \multicolumn{6}{|l|}{ Gender } \\
\hline \multirow[t]{2}{*}{ Female } & Arg/Arg & 5 & 3 & & \\
\hline & Arg/Pro + Pro/Pro & 42 & 43 & 0.74 & $0.59(0.13-2.61)$ \\
\hline \multirow[t]{2}{*}{ Male } & Arg/Arg & 58 & 62 & & \\
\hline & Arg/Pro + Pro/Pro & 237 & 239 & 0.78 & $1.06(0.71-1.58)$ \\
\hline \multicolumn{6}{|l|}{$\begin{array}{l}\text { Hepatitis B } \\
\text { surface antigen }\end{array}$} \\
\hline \multirow[t]{2}{*}{-} & Arg/Arg & 31 & 45 & & \\
\hline & Arg/Pro + Pro/Pro & 127 & 162 & 0.62 & $1.14(0.68-1.90)$ \\
\hline \multirow[t]{2}{*}{+} & Arg/Arg & 32 & 20 & & \\
\hline & Arg/Pro + Pro/Pro & 152 & 120 & 0.45 & $0.79(0.43-1.45)$ \\
\hline
\end{tabular}

HCC, hepatocellular carcinoma; OR, odds ratio; CI, confidence interval; Arg, arginine; Pro, proline.

Table IV. Interaction analysis of the p53 codon 72 polymorphism with smoking, alcohol consumption, gender and HBsAg.

\begin{tabular}{|c|c|c|c|c|}
\hline Characteristics & $\mathrm{HCC}$ & Control & P-value & OR $(95 \% \mathrm{CI})$ \\
\hline Smoking(-) + Arg/Arg & 33 & 55 & & \\
\hline Smoking(-) + Arg/Pro + Pro/Pro & 146 & 171 & 0.15 & $1.42(0.88-2.31)$ \\
\hline $\operatorname{Smoking}(+)+\operatorname{Arg} / \operatorname{Arg}$ & 30 & 10 & $0.00^{\mathrm{a}}$ & $5.00(2.17-11.53)$ \\
\hline Smoking $(+)+$ Arg/Pro + Pro/Pro & 133 & 111 & $0.00^{\mathrm{a}}$ & $2.00(1.21-3.29)$ \\
\hline Drinking(-) + Arg/Arg & 26 & 36 & & \\
\hline Drinking(-) + Arg/Pro + Pro/Pro & 110 & 157 & 0.92 & $0.97(0.55-1.70)$ \\
\hline $\operatorname{Drinking}(+)+\operatorname{Arg} / \operatorname{Arg}$ & 37 & 29 & 0.11 & $1.77(0.88-3.56)$ \\
\hline Drinking $(+)+$ Arg/Pro + Pro/Pro & 169 & 125 & $0.03^{\mathrm{a}}$ & $1.87(1.08-3.26)$ \\
\hline Female + Arg/Arg & 5 & 3 & & \\
\hline Female + Arg/Pro + Pro/Pro & 42 & 43 & 0.74 & $0.59(0.13-2.61)$ \\
\hline Male + Arg/Arg & 58 & 62 & 0.68 & $0.56(0.13-2.46)$ \\
\hline Male + Arg/Pro + Pro/Pro & 237 & 239 & 0.72 & $0.60(0.14-2.52)$ \\
\hline $\operatorname{HBsAg}(-)+\operatorname{Arg} / \operatorname{Arg}$ & 31 & 45 & & \\
\hline HBsAg(-) + Arg/Pro + Pro/Pro & 127 & 162 & 0.62 & $1.14(0.68-1.90)$ \\
\hline $\operatorname{HBsAg}(+)+\operatorname{Arg} / \operatorname{Arg}$ & 32 & 20 & $0.02^{\mathrm{a}}$ & $2.32(1.13-4.78)$ \\
\hline HBsAg $(+)+\operatorname{Arg} /$ Pro + Pro/Pro & 152 & 120 & $0.02^{\mathrm{a}}$ & $1.84(1.10-3.08)$ \\
\hline
\end{tabular}

${ }^{a} \mathrm{P}<0.05$. HBsAg, hepatitis B surface antigen; HCC, hepatocellular carcinoma; OR, odds ratio; CI, confidence interval; Arg, arginine; Pro, proline.

that was investigated. The results of the current study indicated no significant association between the p53 codon 72 polymorphism and susceptibility to $\mathrm{HCC}$ in a Chinese population from northeast Sichuan (Arg/Pro: OR, 1.00 and 95\% CI, 0.66-1.50; Pro/Pro: OR, 1.06 and 95\% CI, 0.69-1.64; Arg/Pro + Pro/Pro: OR, 1.02 and 95\% CI, 0.70-1.50). 
Furthermore when stratified analyses were performed with smoking, alcohol consumption, gender and HBV infection, no risk-increasing genotype was identified. However, the interaction between p53 codon 72 polymorphism and exposure to the above-mentioned environmental conditions may increase the risk of HCC (smoking OR, 2.00 and 95\% CI, 1.21-3.29; alcohol consumption OR, 1.87 and 95\% CI, 1.08-3.26; HBV infection OR, 1.84 and 95\% CI, 1.10-3.08). The current findings were consistent with the results reported by Zhang et al (9), who did not observe a correlation between the p53 codon 72 polymorphism and risk of HCC in Guangxi, China (9), while $\mathrm{Mu}$ et al (10) identified an association between the p53 codon 72 polymorphism and susceptibility to HCC in Shandong, China (10). Zhu et al (11) did not find an association between $\mathrm{p} 53$ codon 72 polymorphism and the risk of HCC, but confirmed that the Pro allele increased the risk of HCC in HBsAg-negative subjects, and exerted a synergistic influence on the risk of HCC when combined with family history of HCC and the male gender. In addition to China, many research groups have been addressing the same issue in other locations. Ezzikouri et al (12) indicated that p53 codon 72 polymorphism conferred a significantly elevated risk of HCC in a Moroccan population and Sümbül et al (13) suggested the Pro/Pro homozygote may be a genetic susceptibility factor for HCC in a Turkish population, particularly in males and HBV-infected patients. In addition, Yoon et al (14) observed that the Pro allele was associated with an increased risk of $\mathrm{HCC}$ in $\mathrm{HBV}$-infected patients in a Korean population. By contrast, Di Vuolo et al (15) proposed that this polymorphism was not a potential risk factor for development of HCC in Italian patients. The discrepancies among these studies may be due to the geographic location of the study subjects, the sample size, statistical methods, confounding factors and genotyping assays. For example, differences in ethnicity may affect the distribution of alleles, in addition to the latitude, temperature and intensity or UV rays (16).

The development of HCC is a multistep process, which is affected by genetic and environmental factors (17). Smoking may be a risk factor for HCC, as the harmful substances contained in tobacco (such as polycyclic aromatic hydrocarbons and nitrosamines) lead to the degeneration, necrosis and regeneration of hepatocytes, as well as the destruction and reconstruction of hepatic lobules, and ultimately induce HCC (18). The current findings indicate that smoking individuals carrying the Pro/Pro genotype had a 2.00-fold increased risk of $\mathrm{HCC}$ when compared with non-smoking individuals with the Arg/Arg genotype.

Previous studies have demonstrated that alcohol consumption is another risk factor for HCC. In the current study, alcohol-consuming individuals with the Pro/Pro genotype had a 1.87-fold increased risk of HCC when compared with those carrying the Arg/Arg genotype that were non-drinkers. As the majority of alcohol is metabolized in the liver, ethanol will be oxidized into aldehyde, which may be a carcinogenic substance that leads to HCC (19).

In addition, the present findings indicated that HBsAg-positive individuals with the Pro/Pro genotype exhibited a 1.84-fold increased risk of HCC when compared with HBsAg-negative carriers with the Arg/Arg genotype. $\mathrm{HBV}$ infection has been confirmed as a major risk factor for
HCC. HBVx is the main functional gene in the HBV genome; the encoded protein weakens nucleotide excision repair (20) by binding with the wild-type p53 protein in the cytoplasm and hindering p53 translocation into the nucleus. Furthermore, Park et al (21) demonstrated that the HBx infection activates $\mathrm{NF}-\kappa \mathrm{B}$ and cyclin D1 to accelerate the process of HCC. Epidemiological surveys have indicated a male to female ratio of 2.78:1 and 2.74:1 for morbidity and mortality of HCC, respectively, indicating an association with hormones (22). Certain scholars hypothesized that estrogen prevents cancer in physiological conditions, inhibits the canceration of hepatic hyperplastic nodules, and suppresses HBV DNA transcription and replication (23). Other studies have confirmed that the levels of interleukin- 6 produced by male mice with liver cancer are higher than in females, and activate signal transducer and activator of transcription (STAT)3 via JNK-STAT signaling pathway, triggering immunosuppression, hepatocyte apoptosis and tissue repair, which eventually leads to HCC (24). While in the current study no link between the p53 codon 72 polymorphism and $\mathrm{HCC}$ was identified following the stratified and interaction analysis by gender, which is inconsistent with the results of previous studies $(12,13)$. The difference may be due to sample size and gender proportion, and further studies based on larger sample size are required to clarify this.

Carcinogenesis is a process of biological system crash caused by alterations of oncogenes, tumor suppressor genes and epigenetic changes; thus a single gene mutation may not significantly increase the susceptibility to HCC. Although in the present study, the p53 codon 72 polymorphism was not associated with the risk of HCC, p53 may interact with other genes, such as mouse double minute 2 homolog, phosphatase and tensin homolog, p14ARF and c-Myc, to increase the risk; however, this requires further investigation.

There were certain limitations of the present study, which must be considered when interpreting the findings. Firstly, the sample size of this research is relatively small, further studies based on a larger number of subjects are required to confirm this issue. Secondly, this was a hospital-based case-control study; therefore, patients were recruited from the same hospital, which may be less representative of HCC patients in the general population. Future multicenter research trials in this area are therefore required. Furthermore, although all of the patients were from the same hospital, the ethnicity and geographic location should have been considered, which may be another factor that influenced the allele frequency. Further investigations are required to validate the information in patients from different regions and ethnic groups.

In conclusion, these results indicate no significant association between the $\mathrm{p} 53$ codon 72 polymorphism and susceptibility to $\mathrm{HCC}$ in a Chinese population from northeast Sichuan. However, interactions between genetic factors and environmental exposure significantly increased the risk of HCC, which suggests that certain environmental exposure controls, such as smoking and drinking cessation, and hepatitis $\mathrm{B}$ vaccination may effectively prevent the development of HCC.

\section{Acknowledgements}

The present study was supported by Sichuan Science and Technology Agency (grant no. 2014JY0115). This abstract was 
presented at the AACR 106th Annual Meeting, April 18-22, 2015 in Philadelphia, PA, USA and was published as Abstract no. 1226 in Cancer Res (Suppl 15).

\section{References}

1. Zhang S,Zheng R, Zeng $\mathrm{H}$ and Chen W: The incidence differences among sex, geographical areas and mean age of diagnosis for liver cancer in China, 1989-2008. Zhonghua Yu Fang Yi Xue Za Zhi 48: 355-360, 2014 (In Chinese).

2. Ma X, Liu XX and Wu XP: Incidence and mortality of cancer in the registered regions of Sichuan in 2009. J Prev Med Inf 29: 918-923, 2013

3. Chen JG, Lu JH, Zhu YR, Zhu J and Zhang YH: A thirty-one year prospective follow-up program on the HBsAg carrier state and primary liver cancer in Qidong, China. Zhonghua Liu Xing Bing Xue Za Zhi 31: 721-726, 2010 (In Chinese).

4. Cheok CF, Verma CS, Baselga J and Lane DP: Translating p53 into the clinic. Nat Rev Clin Oncol 8: 25-37, 2011.

5. Hu S, Zhao L, Yang J and Hu M: The association between polymorphism of P53 Codon72 Arg/Pro and hepatocellular carcinoma susceptibility: Evidence from a meta-analysis of 15 studies with 3,704 cases. Tumour Biol 35: 3647-3656, 2014.

6 . Forner A and Bruix J: Biomarkers for early diagnosis of hepatocellular carcinoma. Lancet Oncol 13: 750-751, 2012.

7. Insinga A, Cicalese A, Faretta M, Gallo B, Albano L, Ronzoni S, Furia L, Viale A and Pelicci PG: DNA damage in stem cells activates $\mathrm{p} 21$, inhibits $\mathrm{p} 53$, and induces symmetric self-renewing divisions. Proc Natl Acad Sci USA 110: 3931-3936, 2013.

8. Leu JI, Murphy ME and George DL: The p53 codon 72 polymorphism modifies the cellular response to inflammatory challenge in the liver. J Liver 2: 117, 2013.

9. Zhang YY, Yu HP and Fan XJ: Association of p53 codon72 polymorphism and risk of hepatocellular carcinoma. J Clin Oncol 18: 189-193, 2012.

10. Mou NN and Zhang L: Effects of p53 gene codon 72 Arg/Pro polymorphisms on susceptibility of hepatocellular carcinoma in patients with hepatitis B virus infection in Shandong Province. J Hepatopancreatobilliary Surg 25: 381-384, 2013.

11. Zhu ZZ, Cong WM, Zhu GS, Liu SF, Xian ZH, Wu WQ, Zhang XZ, Wang YH and Wu MC: Association of p53 codon 72 polymorphism with genetic susceptibility to hepatocellular carcinoma in Chinese population. Zhonghua Yi Xue Yi Chuan Xue Za Zhi 22: 632-635, 2005 (In Chinese).

12. Ezzikouri S, El Feydi AE, Chafik A, Benazzouz M, El Kihal L, Afifi R, Hassar M, Pineau P and Benjelloun S: The Pro variant of the p53 codon 72 polymorphism is associated with hepatocellular carcinoma in Moroccan population. Hepatol Res 37: 748-754, 2007.
13. Sümbül AT, Akkız H, Bayram S, Bekar A, Akgöllü E and Sandıkçı M: p53 codon 72 polymorphism is associated with susceptibility to hepatocellular carcinoma in the Turkish population: A case-control study. Mol Biol Rep 39: 1639-1647, 2012.

14. Yoon YJ, Chang HY, Ahn SH, Kim JK, Park YK, Kang DR, Park JY, Myoung SM, Kim DY, Chon CY, et al: MDM2 and p53 polymorphisms are associated with the development of hepatocellular carcinoma in patients with chronic hepatitis B virus infection. Carcinogenesis 29: 1192-1196, 2008.

15. Di Vuolo V, Buonaguro L, Izzo F, Losito S, Botti G, Buonaguro FM and Tornesello ML: TP53 and MDM2 gene polymorphisms and risk of hepatocellular carcinoma among Italian patients. Infect Agent Cancer 6: 13, 2011.

16. Lu SQ, Jia ST and Luo Y: Recent advances in mutant p53 and novel personalized strategies for cancer therapy. Yi Chuan 33 539-548, 2011 (In Chinese).

17. Lv L, Wang P, Zhou X and Sun B: Association between the $\mathrm{p} 53$ codon 72 Arg/Pro polymorphism and hepatocellular carcinoma risk. Tumour Biol 34: 1451-1459, 2013.

18. Zhang XF, Wei T, Liu XM, Liu C and Lv Y: Impact of cigarette smoking on outcome of hepatocellular carcinoma after surgery in patients with hepatitis B. PLoS One 9: e85077, 2014.

19. Li Y, Yang $\mathrm{H}$ and Cao J: Association between alcohol consumption and cancers in the Chinese population - a systematic review and meta-analysis. PLoS One 6: e18776, 2011.

20. Kapoor NR, Ahuja R, Shukla SK and Kumar V: The HBx protein of hepatitis B virus confers resistance against nucleolar stress and anti-cancer drug-induced p53 expression. FEBS Lett 587: 1287-1292, 2013.

21. Park SG, Min JY, Chung C, Hsieh A and Jung G: Tumor suppressor protein $\mathrm{p} 53$ induces degradation of the oncogenic protein HBx. Cancer Lett 282: 229-237, 2009.

22. Hartwell HJ, Petrosky KY, Fox JG, Horseman ND and Rogers AB: Prolactin prevents hepatocellular carcinoma by restricting innate immune activation of c-Myc in mice. Proc Natl Acad Sci USA 111: 11455-11460, 2014.

23. Keng VW, Largaespada DA and Villanueva A: Why men are at higher risk for hepatocellular carcinoma? J Hepatol 57: 453-454, 2012.

24. Naugler WE, Sakurai T,Kim S, Maeda S, Kim K, Elsharkawy AM and Karin M: Gender disparity in liver cancer due to sex differences in MyD88-dependent IL-6 production. Science 317: 121-124, 2007. 